\title{
Análise de Repositórios de REAs em Relação ao Uso dos Padrões de Linked Open Data
}

\author{
Luciano Bernardes de Paula ${ }^{1}$, William Simão de Deus ${ }^{2}$, Ellen Francine Barbosa ${ }^{2}$ \\ ${ }^{1}$ Instituto Federal de São Paulo (IFSP) - Câmpus Bragança Paulista \\ Av. Major Fernando Valle, 2013 - São Miguel - Bragança Paulista - SP, 12903-000 \\ ${ }^{2}$ Instituto de Ciências Matemáticas e de Computação (ICMC) \\ Universidade de São Paulo (USP) \\ Av. Trab. São Carlense, 400 - Centro, São Carlos - SP, 13566-590 \\ lbernardes@ifsp.edu.br, williamsimao@usp.br, francine@icmc.usp.br
}

\begin{abstract}
Open Educational Resources (OERs) are a very important part of Open Education. These resources are openly shared, allowing their use, reuse, and adaptation. An important aspect to make the search for OERs in repositories on the Web efficient is how the metadata of theses resources should expose data about them. A global initiative to define standards to allow access to open data on the Web is the Linked Open Data (LOD). In this paper it is presented an analysis of repositories of OERs about the use of LOD standards in their metadata. It is also presented a discussion about the results and the advantages of this adoption by the repositories.
\end{abstract}

Resumo. Recursos Educacionais Abertos (REAs) são uma parte muito importante para a Educação Aberta. Esses recursos são compartilhados de forma aberta, possibilitando seu uso, reuso e adaptação livres. Um ponto importante para tornar eficiente a busca por REAs em repositórios pela Web é como o metadado deve expor dados sobre o recurso. Uma iniciativa global para definir padrões para propiciar acesso a dados abertos na Web é o Linked Open Data. Este artigo apresenta uma análise de repositórios de REAs a respeito do uso dos padrões de LOD em seus metadados. Ao final é feita uma discussão sobre os resultados dessa análise e as vantagens dessa adoção pelos repositórios.

\section{Introdução}

É possível afirmar que os Recursos Educacionais Abertos (REAs) são uma importante parte do conceito de Educação Aberta. Segundo [Atkins et al. 2007], REAs são recursos disponibilizados em qualquer mídia, que podem ser utilizados para ensinar, aprender e pesquisar. Esses recursos são publicados sob licença aberta que permite seu uso gratuito, possibilitando que estes sejam readequados para qualquer propósito. Isso inclui cursos completos, materiais de cursos, textos, vídeos, software e quaisquer outras mídias.

Atualmente, muitos desses recursos são digitais e, com a popularização da Web, foram criados os repositórios digitais de REAs, nos quais é possível fazer buscas por recursos sobre um determinado assunto. Para tornar eficiente essa busca via Web, é muito importante o uso de metadados que sejam completos em informações.

$\mathrm{Na}$ Web atual, há uma iniciativa global para definir padrões para compartilhamento de dados abertos, o Linked Open Data (LOD). Por meio dos princípios e padrões do LOD, 
é possível atingir níveis relevantes de compartilhamento de qualquer dado que seja aberto, como é o caso dos REAs, o que propiciaria experiências ricas para aqueles que queiram aprender e pesquisar sobre diversos assuntos ${ }^{1}$.

Existem diversas vantagens de se utilizar padrões do LOD em repositórios de REAs. A utilização desses padrões enriquecem a forma como o metadado dos REAs podem ser interpretados, dando significado às relações que o REA pode ter com outros recursos. O uso desses padrões possibilita também a interligação de recursos a outras bases que, inclusive, podem não ser de REAs. Por exemplo, um professor, ao buscar por REAs sobre uma região do Brasil pode, por meio das ligações no metadado dos recursos encontrados, chegar até os sites oficiais das prefeituras das capitais dos estados da região pesquisada, ou até mesmo em uma base de dados geográficos, onde poderá obter informações sobre o relevo e clima daquela região. Caso todos esses recursos estejam interligados via metadado e utilizando os padrões LOD, todo processo se torna transparente para o usuário, sendo executado pelo próprio sistema de busca, uma vez que os dados são bem estruturados em formatos legíveis por máquinas ${ }^{2}$.

No entanto, esta área ainda é vagamente explorada. Ao revisar a literatura sobre o tema, [Nahhas et al. 2018] declararam que as abordagens existentes encontram-se de modo disperso, sem objetivos claros que possam ajudar a entender o impacto de conceitos do Linked Data ${ }^{3}$ para os REAs. Ou seja, esse potencial ainda não é explorado.

Dessa forma, este artigo tem como objetivo apresentar uma análise sobre o atual nível de uso dos padrões de LOD em repositórios de REAs e apresentar as vantagens que a utilização desses padrões propicia nesse contexto. Para isso foi feita uma análise de repositórios conhecidos e buscas no site Linked Open Data Cloud ${ }^{4}$ por repositórios de REAs. Este trabalho encontra-se estruturado da seguinte forma: na Seção 2 é apresentada uma síntese dos trabalhos relacionados com este; na Seção 3 é apresentado o que são repositórios de REAs e sua relação com metadados; na Seção 4 é apresentado o que é LOD e seus padrões e a classificação de cinco estrelas para bases de dados; na Seção 5 é apresentada a metodologia utilizada na análise dos repositórios; na Seção 6 são apresentados os resultados encontrados; na Seção 7 é apresentada uma discussão sobre os resultados, impactos e limitações do trabalho; a Seção 8 concentra as considerações finais do estudo.

\section{Trabalhos relacionados}

Foram encontrados vários trabalhos que investigam tópicos discutidos neste artigo. Entretanto, uma limitação comumente encontrada nesses trabalhos foi a ausência de uma análise sobre o uso de LOD em repositórios de REAs. Os trabalhos que mais se relacionam com este visam o uso dos padrões de LOD para melhorar a interoperabilidade de REAs em repositórios. Por exemplo, um dos problemas relatados na literatura a respeito da interoperabilidade entre repositórios de REAs é a falta de padronização dos metadados. Em [Koutsomitropoulos and Solomou 2018] foi apresentada a possibilidade de se utilizar ontologias, que são utilizadas em LOD, para facilitar a interligação de bases de dados.

\footnotetext{
${ }^{1}$ Benefícios do LOD - https://www.w3.org/2005/Incubator/lld/wiki/Benefits - acessado em 28/09/2020.

${ }^{2} \mathrm{~A}$ apresentação de detalhes técnicos sobre esse processo estão além do escopo deste trabalho.

${ }^{3}$ Linked Data considera não só os dados abertos, mas qualquer tipo de dado.

${ }^{4}$ LOD Cloud - site que possui informações sobre o atual estado do LOD em relação a bases interligadas - https://lod-cloud.net/ - acessado em 28/09/2020.
} 
[Rajabi et al. 2014] apresentaram como expor metadados seguindo o padrão IEEE $\mathrm{LOM}^{5}$. Para isso, foi feita uma relação entre esse metadado e os padrões do LOD. Nesse sentido, nota-se que as generalizações dos resultados foram restritas somente aos repositórios que adotam o padrão IEEE LOM.

Em [Sicilia et al. 2010] foi apresentado um exemplo prático de um portal de repositórios de REAs interligado utilizando LOD, apresentando as vantagens que essa ação propicia. Em [Kawase et al. 2013] foi abordada a questão de outra forma, apresentando como foi construída uma plataforma que coleta dados sobre objetos de aprendizagem, tratando a heterogeneidade com o uso de LOD. Em ambos os trabalhos, no entanto, notou-se a limitação dos acervos e o total de áreas analisadas. Complementarmente, [Navarrete and Luján-Mora 2015] realizou uma contribuição teórica sobre as vantagens de se usar LOD em repositórios de REA.

[Vega-gorgojo et al. 2015] conduziu uma revisão sistemática da literatura a respeito de trabalhos que unem o conceito de LOD com aprendizagem e objetos educacionais. O trabalho também apresentou recomendações a respeito do uso dos padrões de LOD em repositórios. Dentre os estudos citados, não há nenhum que faz uma análise dos repositórios de REAs em relação ao uso dos padrões de LOD.

Por fim, [Lama et al. 2011] apresentou um sistema automatizado para interligar um repositório de REAs com a DBpedia, um portal que possui os metadados dos artigos da Wikipedia. Este trabalho não considerou a interligação do repositório com outros.

\section{Repositórios de REAs e metadados}

Atualmente existem diversos repositórios de REAs disponíveis na Web. Esses repositórios possibilitam que os REAs sejam acessados via Web, pesquisados por assunto e palavraschave, independente se o recurso é um texto, um vídeo, uma imagem, etc.

Uma parte importante a ser considerada para os repositórios de REAs são os metadados relacionados com os recursos armazenados no repositório. Um metadado pode ser traduzido como um dado sobre outro dado [Valiente et al. 2015]. Por exemplo, um arquivo de vídeo digital pode possuir um arquivo metadado que explica o seu conteúdo, por meio de informações como título, autor, assunto, tipo de mídia, etc. A função do metadado em um REA é de grande importância, pois possibilita a obtenção de informações sobre o recurso sem que seja necessário visualizá-lo por completo. Como no exemplo anterior, um REA em forma de vídeo, sem metadados adequados, faz com que haja a necessidade do usuário assisti-lo para entender quais assuntos são abordados no recurso.

Tudo isso é ainda mais importante ao se considerar a busca por REAs de forma automatizada, executada por máquinas. Uma simples busca por palavras-chave em um repositório de REA é um exemplo de busca automatizada e essa possuirá resultados melhores caso o metadado dos recursos sejam ricos e completos em informações.

\section{Linked Open Data - LOD}

Tim Berners-Lee, considerado o criador da Web, definiu o conceito de dados interligados (linked $\left.d a t a^{6}\right)$. Esse conceito é baseado em quatro princípios: i) uso de URIs (Uniform

\footnotetext{
${ }^{5}$ IEEE LOM - https://bit.ly/347dxNh - acessado em 28/09/2020

${ }^{6}$ Linked Data - https://www.w3.org/DesignIssues/LinkedData.html - acessado em 28/09/2020.
} 
Resource Identifier) para dar nomes a recursos publicados na Web; ii) uso de URIs via HTTP (Hypertext Transfer Protocol) para que esses nomes possam ser consultados na Web; iii) ao acessar um URI, deve ser provido informações úteis, utilizando RDF (Resource Description Framework) e SPARQL (uma linguagem de busca em bases de dados de arquivos RDF).; iv) é preciso incluir links para outros URIs, dessa forma é possível descobrir novos recursos.

Considerando dados abertos, ou seja, aqueles que podem ser acessados, consumidos e reutilizados de forma livre, Tim Berners-Lee também definiu o conceito de dados abertos interligados, ou Linked Open Data (LOD), que tem como objetivo interligar dados abertos, formando uma rede de metadados que conecta conceitos e recursos.

\subsection{Cinco estrelas do LOD}

Tim Berners-Lee elaborou uma classificação de cinco estrelas, acumulativas, para aferir quanto um dado está disponibilizado seguindo os padrões de $\mathrm{LOD}^{7}$. Essa classificação inclui o uso de licenças públicas e considera que os dados são publicados na Web. Ao se utilizar os padrões e publicar os dados sob uma licença aberta, pode-se considerar que o padrão de LOD está sendo usado. É possível que organizações que não possuam dados abertos também utilizem essa classificação, entretanto, é dito que seguem os padrões de Linked Data (LD). Uma vez que este artigo considera o uso desses padrões para REAs, que são essencialmente abertos, esta pesquisa considerou o uso dos padrões de LOD.

O significado da classificação de 1 a 5 estrelas é apresentado na Tabela 1.

\begin{tabular}{|c|l|}
\hline Estrelas & Descrição \\
\hline$\star$ & Dados publicados na Web em qualquer formato sob uma licença aberta. \\
\hline$\star \star$ & Dados publicados de forma estruturada e em um formato que seja legível por máquinas. \\
\hline$\star \star \star$ & Dados publicados de forma estruturada na Web e utilizando um formato não-proprietário. \\
\hline$\star \star \star \star$ & Dados publicados na Web no formato RDF. \\
\hline$\star \star \star \star \star$ & O metadado no formato RDF indica URIs de outras bases de dados relacionadas. \\
\hline
\end{tabular}

Tabela 1. Descrição da classificação de 5 estrelas do LOD.

\subsection{Padrões de LOD}

O LOD indica como padrão para metadados o framework RDF. Por meio deste, é possível descrever um recurso em forma de triplas do tipo $<$ sujeito $><$ predicado $><$ objeto $>^{8}$. O RDF pode ser expressado em diversos formatos compatíveis como, por exemplo, Turtle, $\mathrm{N}$-triples, $\mathrm{N}$-quads, RDF/XML, entre outros.

O metadado em RDF de um recurso pode ser disponibilizado de diversas formas: em um arquivo RDF separado; em anotações embarcadas no código HTML da página, seguindo o padrão RDFa; exposto em um endpoint SPARQL, ou até mesmo na forma de dataset com os metadados para download. Há também vocabulários que podem ser expressados utilizando RDF/XML ou equivalente, como é o caso do Dublin Core9

\footnotetext{
75 Star Linked Open Data - https://dvcs.w3.org/hg/gld/raw-file/default/glossary/index.html\#x5-starlinked-open-data - acessado em 28/09/2020.

${ }^{8}$ Por exemplo, uma página em uma URL $U_{x}$ pode ser a respeito de um assunto $A_{y}$, portanto, uma tripla genérica poderia ser $<U_{x}><$ sobre $><A_{y}>$, seguindo a ordem $<$ sujeito $><$ predicado $><$ objeto $>$ e cada uma das três partes são, por sua vez, também URIs.

${ }^{9}$ Dublin Core - https://www.dublincore.org/ - acessado em 28/09/2020.
} 
IX Congresso Brasileiro de Informática na Educação (CBIE 2020)

Anais do XXXI Simpósio Brasileiro de Informática na Educação (SBIE 2020)

\section{Metodologia}

Para atingir o objetivo proposto neste trabalho, que é analisar o nível do uso dos padrões de LOD nos principais repositórios de REAs, apresentando as vantagens dessa adoção, foram desenvolvidas duas etapas distintas de pesquisa:

- Etapa 1: análise de repositórios de REAs com o intuito de verificar se tais repositórios utilizam os padrões do LOD e como é feito esse uso.

- Etapa 2: análise dos repositórios de REAs encontrados por meio de buscas no site Linked Open Data Cloud. Esta etapa teve como intuito verificar se há repositórios de REAs listados na nuvem do LOD, mesmo que não sejam conhecidos.

A seguir, o detalhamento das ações realizadas em cada etapa é apresentado.

\subsection{Método usado para análise dos repositórios}

$\mathrm{Na}$ Etapa 1, os repositórios foram acessados e tiveram suas coleções e estruturas analisadas. Durante essa etapa, as seguintes informações foram observadas:

- Como são compartilhados os metadados dos REAs disponíveis no repositório?

- Como os repositórios estão utilizando padrões recomendados pelo LOD?

Para extrair essas informações, foi realizado o seguinte procedimento: i) busca manual pelo metadado do REA encontrado no repositório, caso este metadado seja disponibilizado em um arquivo separado; ii) utilização de dois extratores automáticos online de metadado descritos usando RDF/RDFa e outros padrões relacionados. Os extratores utilizados foram: RDFa 1.1 Distiller and Parser ${ }^{10}$ e Graphite Browser ${ }^{11}$. Esses dois extratores funcionam de forma equivalente: por meio de sua página principal, é possível inserir um URL e os esses apresentam as triplas encontradas embarcadas na página encontrada no endereço ou mesmo em arquivo separado, residente no mesmo endereço da página; iii) busca manual no código HTML da página de apresentação do REA por termos chave que indiquem metadado em RDF/RDFa e outros padrões relacionados. Neste caso, foi analisado o código fonte da página, buscando termos como "rdf", entre outros, na tentativa de se visualizar se há anotações relacionadas com metadado.

É importante citar que em relação aos metadados reconhecidos pelos extratores automáticos foi feita a conferência dos resultados obtidos, pois em alguns casos esses não eram relacionadas às características específicas do REA em si, mas de informações genéricas sobre o recurso (por exemplo: somente título e tipo de mídia) e/ou sobre a página do repositório. Dessa forma, o resultado dos extratores foi validado para certificação de que o metadado obtido era ou não sobre característica relevantes do REA.

A partir das informações obtidas, cada repositório foi classificado de acordo com as classificação apresentada na Seção 4.1. Os repositórios considerados na Etapa 1 foram: MIT OpenCourseWare, OER Commons, EduCapes, Repositório Aberto, MetaFinder, SkillsCommon, Merlot, Xpert, iOER e OAsis.

Na Etapa 2 foram feitas buscas no site Linked Open Data Cloud por dados sobre REAs. Para isso, foram testados diferentes termos de busca, como mostrado a seguir:

\footnotetext{
${ }^{10}$ RDFa 1.1 Distiller - https://www.w3.org/2012/pyRdfa/ - acessado em 28/09/2020.

${ }^{11}$ Graphite Browser - http://graphite.ecs.soton.ac.uk/browser/ - acessado em 28/09/2020.
} 
- Inicialmente, foi feita uma busca pelos termos educational resources, o que resultou em 4 bases.

- Então foi realizada uma busca com o termo educational. Foram obtidos 17 resultados, sendo que os 4 resultados da consulta anterior estavam contidos nesses.

- Tornando a busca ainda mais abrangente, foi feita uma consulta utilizando o termo education. Dessa vez, foram obtidos 28 resultados, sendo que os 17 resultados da pesquisa anterior estavam contidos dentre esses. Foi estabelecido, portanto, que esse seria o resultado investigado.

Ao fim, foram analisadas as 28 bases resultantes, na tentativa de se entender quais delas possuem, de fato, relação com REAs. Essa análise resultou em 6 bases. É importante citar que uma das bases resultantes nesta última consulta não foi obtida em nenhuma das duas consultas anteriores, o que valida a estratégia de se obter resultados mais amplos. A lista com os repositórios selecionados é apresentado na Tabela $2^{12}$.

\begin{tabular}{|l|l|c|}
\hline Repositório & Links & Etapa \\
\hline MIT OpenCourseWare & https://ocw.mit.edu/ & 1 \\
\hline OER Commons & http://oercommons.org/ & 1 \\
\hline EduCapes & https://educapes.capes.gov.br/ & 1 \\
\hline Repositório Aberto & https://repositorioaberto.uab.pt/ & 1 \\
\hline Mason OER MetaFinder & https://oer.deepwebaccess.com/oer/desktop/en/search.html & 1 \\
\hline SkillsCommons & https://skillscommons.org & 1 \\
\hline Merlot & https://www.merlot.org/merlot/ & 1 \\
\hline Xpert & https://www.nottingham.ac.uk/xpert/ & 1 \\
\hline OAsis & http://oasis.col.org/ & 1 \\
\hline iOER & http://ioer.ilsharedlearning.org/ & 1 \\
\hline Dados abertos da Open University & https://data.open.ac.uk & 2 \\
\hline Didactalia & http://didactalia.net/ & 2 \\
\hline IneveryCREA & http://ineverycrea.com/ & 2 \\
\hline IneveryCREA Argentina & http://ineverycrea.com.ar & 2 \\
\hline mEducator & http://meducator.open.ac.uk/ & 2 \\
\hline Organic Edunet Linked Open Data & http://data.organic-edunet.eu/ & 2 \\
\hline
\end{tabular}

Tabela 2. Repositórios analisados nas Etapas 1 e 2.

\section{Resultados}

Nesta seção os resultados obtidos nas Etapas 1 e 2 são detalhados.

\subsection{Resultado da análise dos principais repositórios (Etapa 1)}

A seguir, é apresentada uma descrição das notas definidas para cada tipo de repositório, bem como uma justificativa para essa classificação. É importante citar que o formato PDF (Portable Document Format) foi considerado, neste trabalho, como legível por máquinas (machine-readable). Embora exista essa questão na literatura, atualmente há estudos que já consideram o uso deste formato para esse fim, dada sua grande adoção ${ }^{13}$.

MIT OpenCourseWare: $\star \star \star$ Este repositório é focado em cursos, de diversas áreas, e os recursos derivados desses cursos são disponibilizados como REAs. Não foi

\footnotetext{
${ }^{12}$ Links acessados em 01/08/2020.

${ }^{13}$ Open Data Institute study shows role for PDF with Open Data - https://theblog.adobe.com/open-datainstitute-study-shows-role-for-pdf-with-open-data/ - acessado em 28/09/2020.
} 
encontrado nenhum tipo de metadado específico em relação aos recursos disponibilizados, sendo que as características dos REAs disponibilizados, como título, autor, etc, somente são apresentados em suas respectivas páginas, dentro do repositório. Não foi possível encontrar dataset ou endpoint SPARQL para consulta. Dessa forma, esse repositório possui 3 estrelas na classificação do LOD, uma vez que possui recursos publicados sob licença aberta, em formato legível por máquinas e não proprietário (PDF, por exemplo), porém, não utiliza os padrões do LOD e não interliga seus metadados com outras bases.

OER Commons: $\star \star \star$ Neste repositório foi possível encontrar uma tabela no formato ".xlsx" que descreve os campos utilizados no metadado de seus REAs, assim como o vocabulário utilizado, porém, não são utilizados padrões de LOD. Não foi possível encontrar nenhum dataset dos metadados disponível para download. Este repositório possui um endpoint para acesso a uma base de metadados, porém para acessar o endpoint, é necessário o pagamento de licença para obter um token de autenticação ${ }^{14}$. Dessa forma, é possível considerar que esse repositório possui 3 estrelas na classificação do LOD, uma vez que possui recursos publicados sob licença aberta, em formato legível por máquinas e não proprietário. Porém, não utiliza os padrões do LOD e não há interligação dos metadados com outras bases, uma vez que usa formato e vocabulário próprios.

EduCapes: $\star \star \star$ Neste repositório foi possível identificar que, para cada REA apresentado, há metadado no padrão Dublin Core disponível, de forma embarcada na página que apresenta o recurso. Porém, é interessante citar que, manualmente, no HTML da página do REA, há mais informações a respeito do recurso, em forma de metadado, do que as reconhecidas pelos extratores automáticos. É provável que isso se deve a algum problema de formatação das páginas em relação às anotações, o que impossibilita o reconhecimento por ferramentas automáticas. Não foram encontradas formas de se consultar a base de metadados, seja para download, seja como endpoint SPARQL para consulta. No repositório, há REAs disponibilizados em formato legível por máquinas (PDF), utilizando licenças abertas. Dessa forma, esse repositório é classificado com 3 estrelas.

Repositório Aberto: $\star \star \star \mathrm{O}$ metadado dos REAs são disponibilizados seguindo o padrão Dublin Core, porém, ocorreu a mesma situação que o repositório EduCapes: manualmente, no código HTML da página do REA, há mais informações em forma de metadado, do que as reconhecidas pelos extratores automáticos. É provável que os dois repositórios utilizem a mesma plataforma de software para publicação dos REAs, por isso existe a mesma inconsistência. Não foram encontradas formas de se consultar a base de metadados, seja para download, seja como endpoint para consulta. No repositório, há REAs disponibilizados em formato legível por máquinas (PDF), utilizando licenças abertas. Dessa forma, este repositório também pode ser classificado com 3 estrelas.

OAsis: $\star \star \star$ Os REAs encontrados neste repositório possuem metadado seguindo o padrão Dublin Core embarcado na página HTML. Não foi encontrado endpoint SPARQL ou busca pelo metadado. Assim como o EduCapes e o Repositório Aberto, há mais metadado encontrado manualmente do que o reconhecido pelos extratores. É possível que haja a mesma situação em relação ao formato das páginas que os outros repositórios citados. Os REAs são disponibilizados em PDF e, dessa forma, esse repositório recebe 3 estrelas.

\footnotetext{
${ }^{14}$ Endpoint do OERCommons - https://www.oercommons.org/api/search - é requisitado um token de autenticação para continuar - acessado em 28/09/2020.
} 
SkillCommons: $\star$ Neste repositório, não foram encontrados metadados específicos para os REAs manualmente ou com o auxílio dos extratores. O formato dos REAs disponibilizados eram, em sua maioria em formato proprietário (por exemplo ".doc") ou comprimido (por exemplo ".zip"). Não foi possível encontrar metadado específico sobre o REA tanto manualmente quanto utilizando os extratores. Dessa forma, esse repositório recebe apenas 1 estrela.

MetaFinder, Merlot, Xpert e iEOR: não se aplica Tratam-se de um portais que agregam informações sobre outros repositórios de REAs. Dessa forma, esses portais não possuem nenhum tipo de metadado específico para os REAs encontrados, pois somente redirecionam o usuário para o repositório original. O uso ou não dos padrões de LOD dependem do repositório original do recurso.

\subsection{Resultado dos repositórios encontrados no LOD Cloud (Etapa 2)}

Nesta etapa, foram feitas buscas no site Linked Open Data Cloud em busca de bases relacionadas com REAs. É importante citar que foram consideradas todas as bases relacionadas com REAs e metadados, não necessariamente aquelas que são repositórios dos recursos, de fato. A síntese dos resultados é apresentada a seguir:

Dados abertos da Open University: $\star \star \star \star \star$ Neste repositório são compartilhados os metadados dos REAs disponibilizados pela Open University. São dados de publicações, cursos e materiais multimídia produzidos pela universidade. Todos os recursos são disponibilizados seguindo os padrões de LOD e são, na grande maioria, compartilhados sob licença aberta. Os metadados são disponibilizados nos formatos CSV, JSON, XML e há um endpoint SPARQL para consulta.

Didactalia: $\star \star \star \star \star$ O Didactalia é uma comunidade global e um repositório que proporciona a professores e estudantes a oportunidade de criar, compartilhar e encontrar REAs. É citado que o uso dos padrões de Linked Open Data e a criação de um ecossistema de comunidades de aprendizagem no contexto de aprendizado social são o conceito central do Didactalia. Cada recurso possui um arquivo RDF específico, que pode ser obtido na sua própria página, rico em triplas com várias informações sobre o próprio recurso.

IneveryCREA e IneveryCREA Argentina: $\star \star \star \star \star$ IneveryCREA é uma comunidade de professores e profissionais da educação que tem como objetivo a criação, o desenvolvimento e o compartilhamento de conteúdos e REAs na Espanha e na Argentina. Cada página HTML que disponibiliza um conteúdo possui uma versão com metadados embarcados, relacionados ao recurso apresentado.

mEducator: $\star \star \star \star \star$ O mEducator publica dados sobre repositórios outros repositórios de REAs. Usa um RDF schema para interligar recursos educacionais com datasets da nuvem do LOD. Portanto não se trata de um repositório, mas sim um local para encontrar links para datasets de metadados de repositórios de REAs. Durante a concepção deste trabalho, o endpoint para consulta se encontrava offline e a última atualização em sua seção de notícias foi feita em 2013.

Organic Edunet Linked Open Data: $\star \star \star \star \star \star$ Esse repositório contém metadados, que utilizam os padrões de LOD, de uma coleção de REAs disponibilizados pelo portal Organic.Edunet. Os tópicos incluem agricultura orgânica e agroecologia. 
IX Congresso Brasileiro de Informática na Educação (CBIE 2020)

Anais do XXXI Simpósio Brasileiro de Informática na Educação (SBIE 2020)

\section{Discussão}

Como apresentado, praticamente todos os repositórios analisados na Etapa 1 receberam 3 estrelas na classificação de LOD. Isso se deve ao fato de nenhum deles apresentarem os metadados dos REAs disponíveis utilizando padrões do LOD, e somente disponibilizando os recursos de forma isolada. Em uma avaliação ainda mais rígida, alguns deles, inclusive, receberiam uma estrela a menos, uma vez que o formato utilizado para disponibilização dos recursos educacionais é o formato PDF, até recentemente não indicado para compartilhamento de dados abertos.

Um ponto importante a ser explorado é que muitos REAs podem ser arquivos de vídeo e/ou áudio, que naturalmente não são padrões estruturados nem legível por máquinas, do ponto de vista do LOD. Nesta situação, o papel do metadado é ainda mais relevante. Como pôde ser observado, a maioria dos repositórios analisados possuem pouca informação disponível via metadados.

Nota-se, então, que os repositórios de REAs possuem um amplo caminho a percorrem para serem considerados "abertos" sob o ponto de vista de LOD. Como apresentado, muitos repositórios não possuem mecanismos que facilitam o compartilhamento e acesso aos metadados de seu acervo, como endpoints para consulta aos metadados. Indiretamente, isso faz com que cada repositório tenha uma coleção restrita e fechada em si. Logo, agregadores ou buscadores descentralizados de REAs encontram dificuldades para acessar este acervo e disponibilizá-lo para os usuários.

O uso dos padrões de LOD e as interligações entre repositórios e outras bases auxiliaria na disseminação do uso dos REAs, aumentando sua abrangência e potencial de (re)utilização. Essas interligações naturalmente criariam subclouds dentro da nuvem do LOD, aumentando a interoperabilidade e visibilidade dos repositórios e recursos.

\subsection{Limitações do trabalho}

Uma vez que a definição de REA é bem ampla, o mesmo ocorre com a definição de repositório de REA. Dessa forma, há repositórios que armazenam e compartilham REAs e seus metadados, há aqueles que são portais para REAs disponibilizados em outros repositórios, e há casos híbridos. Esse fato faz com que, apesar de existir exemplares de cada um desses tipos nos repositórios analisados neste trabalho, esses representam apenas uma fração dos repositórios atuais e, dessa forma, os resultados não podem ser generalizados para todos os repositórios existentes, sendo necessário a análise individual de cada um.

\section{Conclusões}

Neste trabalho foi apresentada uma análise do uso de padrões de LOD em repositórios de REAs. O uso desses padrões abrem inúmeras possibilidades de se obter informações ricas sobre o assunto apresentado no REA, propiciando uma busca completa sobre o recurso, inclusive entre bases de dados distintas. Nos resultados, foi possível verificar que a maioria dos repositórios mais conhecidos não utilizam esses padrões ou, quando utilizam, os utilizam de forma superficial, fornecendo somente algumas anotações semânticas gerais sobre o recurso compartilhado, em vez de disponibilizar informações mais detalhadas via endpoint SPARQL, por exemplo. Por outro lado, os repositórios encontrados via LOD Cloud fazem um bom trabalho a esse respeito, porém ainda são em número reduzido. 
Como trabalhos futuros pretende-se prosseguir na exploração dos resultados apresentados neste estudo, assim como todo o potencial que o LOD propicia para repositórios de REAs e para a disseminação desses recursos dentro da nuvem do LOD.

\section{Agradecimentos}

Este estudo foi parcialmente financiado pela Coordenação de Aperfeiçoamento de Pessoal de Nível Superior - Brasil (CAPES) - Código Financeiro 001 e pela Fundação de Amparo à Pesquisa do Estado de São Paulo (FAPESP 2018/26636-2).

\section{Referências}

Atkins, D. E., Brown, J. S., and Hammong, A. L. (2007). A review of the open educational resources (OER) movement: Achievements, challenges, and new opportunities. Creative common, online.

Kawase, R., Fisichella, M., Niemann, K., Pitsilis, V., Vidalis, A., Holtkamp, P., and Pereira Nunes, B. (2013). Openscout: Harvesting business and management learning objects from the web of data. pages 445-450.

Koutsomitropoulos, D. A. and Solomou, G. D. (2018). A learning object ontology repository to support annotation and discovery of educational resources using semantic thesauri. IFLA Journal, 44(1):4-22.

Lama, M., Vidal, J. C., Garcia, E. O., Bugarin, A., and Barro, S. (2011). Semantic linking of a learning object repository to dbpedia. In 2011 IEEE 11th International Conference on Advanced Learning Technologies, pages 460-464.

Nahhas, S., Bamasag, O., Khemakhem, M., and Bajnaid, N. (2018). Added values of linked data in education: A survey and roadmap. Computers, 7:45.

Navarrete, R. and Luján-Mora, S. (2015). Use of linked data to enhance open educational resources. In 2015 International Conference on Information Technology Based Higher Education and Training (ITHET), pages 1-6.

Rajabi, E., Miguel-Angel, S., and Salvador, S.-A. (2014). Interlinking educational resources to web of data through ieee lom. Computer Science and Information Systems.

Sicilia, M., Ebner, H., Sánchez-Alonso, S., Álvarez, F., Abián, A., and Barriocanal, E. (2010). Navigating learning resources through linked data: a preliminary report on the re-design of organic.edunet. volume 717 .

Valiente, M.-C., Sicilia, M.-A., Garcia-Barriocanal, E., and Rajabi, E. (2015). Adopting the metadata approach to improve the search and analysis of educational resources for online learning. Computers in Human Behavior, 51:1134 - 1141. Computing for Human Learning, Behaviour and Collaboration in the Social and Mobile Network Era.

Vega-gorgojo, G., Asensio-Pérez, J., Gómez-Sánchez, E., Bote-Lorenzo, M., MuñozCristóbal, J., and Ruiz-Calleja, A. (2015). A review of linked data proposals in the learning domain. Journal of Universal Computer Science, 21:326-364. 